\title{
Cataracts in corvids: health and welfare implications of lens opacification in a colony of western scrub- jays (Aphelocoma californica)
}

\begin{abstract}
The development of cataracts in a colony of 55 Western scrub jays ((Aphelocoma californica) kept in a colony is reported, etiopathology discussed and welfare implications debated. Birds were examined with direct and indirect ophthalmoscopy and slit lamp biomicroscopy. Intraocular pressures were measured with a rebound tonometer. Thirty eight birds were diagnosed with some lens opacification, ranging from linear arrangements of small vacuoles to mature cataract. In some birds this lens pathology was seen concurrent with adnexal post-traumatic pathology or with substantial uveitic changes. Behaviour changes were only noted in birds with mature or near-mature cataracts. The lack of significant ultraviolet light and the provision of a suitable avian diet suggest that neither excess light nor inadequate nutrition could be factors in cataractogenesis. While a genetic cause is difficult to exclude, the fact that these were wild caught birds from an outbred population without observed lens pathology in the wild renders this unlikely. We suggest that a post-traumatic aetiology is the likely causative factor in the genesis of lens opacities in these birds. The changes in behaviour and the impact on the welfare of birds with these ocular changes are discussed.
\end{abstract}

Volume 2 Issue I - 2017

\author{
David LWilliams, Nigel Salter \\ Department of Veterinary Medicine, University of Cambridge, \\ UK
}

Correspondence: David LWilliams, Department of Veterinary Medicine, University of Cambridge, Madingley Road, Cambridge CB3 0ES UK, Email dlw33@cam.ac.uk

Received: May 10, 2017 | Published: June 12, 2017

\section{Introduction}

The cognitive capacity of avian species has long been underrated; the very phrase 'bird-brained' suggests a pretty minimal executive function in these animals. Yet studies from a small number of investigators - one might particularly highlight Pepperberg's work with African grey parrots ${ }^{1}$ or Clayton's studies with scrub-jays ${ }^{2}$ suggest that at least in psittaciformes and corvidae we see species with considerable cognitive abilities. Indeed Emery, working with Clayton, has termed her birds 'feathered apes' because of their superlative decision-making abilities. ${ }^{3}$ Such suggestions may have significant implications for keeping these birds in an experimental setting. Here we report the development of cataracts in a majority of scrub jays kept in an experimental colony and discuss the possible etiologies for lens opacification in these birds.

Professor Clayton first noted interesting behavioral characteristics of the Western scrub jay (Aphelocoma californica) while undertaking her doctoral studies in the University of California, Davis two decades ago. These birds hide or 'cache' food items for retrieval later but other scrub jays often steal the items after viewing the caching behaviour of one of their compatriots. Clayton recognized that some birds, noting a second bird observing them while caching, then re-hid the food item when the observing bird was no longer present. She realized that such behaviour suggests a 'theory of mind' in the caching bird which can in some way comprehend what the observing bird is thinking and planning to do. Research on a colony of Western scrub-jays brought back to the UK to evaluate their cognitive function has resulted in a large number of scientific research publications, of which a few recent ones are. ${ }^{4-6}$ Experiments by Clayton and her group involve a number of different protocols observing how birds behave while caching (Figure 1) and manipulating their surroundings to investigate elements from tool use to forward planning. Caching behaviour is a key part of these birds' life: the birds are continually motivated to continue caching during the trials regardless of their current nutritional status. Hiding food for future retrieval is a central part of the birds' behaviour. Indeed it is evaluating this planning for the future that has formed an important part of recent research on the birds. ${ }^{7}$ Much of this research depends on birds having good visual capability, both for caching itself and also observing birds caching themselves. Birds were, however, noted to be developing cataracts within the first three years of captivity. This report documents the lesions observed and discusses possible etiopathogenesis of the opacities.

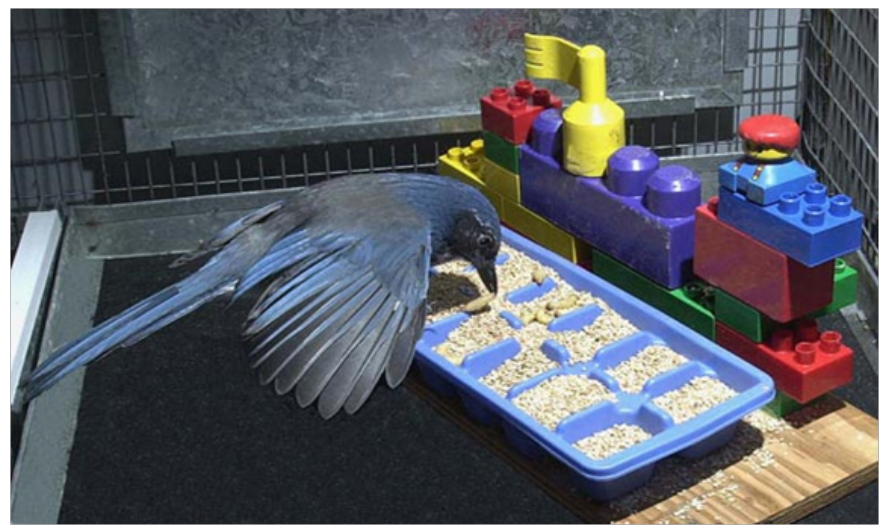

Figure I Scrub jay involved in caching experiment (courtesy Professor NJ Clayton).

Avian cataracts have been widely reported in the veterinary literature with numerous causes postulated or proved. Antiracial pigeons have a lens opacity at hatching associated with glycogen build-up in the lens although this disappears as the glycogen is metabolised. ${ }^{8}$ Congenital cataract has been recorded in ducklings, ${ }^{9}$ while early onset lens opacity with intraocular inflammation has been seen in broiler chickens, ${ }^{10}$ with similar changes recorded in 
bobwhite quail ${ }^{11}$ and a different cataract morphology in Japanese quail with an inherited myotonia ${ }^{12}$ Presumed inherited cataract was noted by six months of age in Brahama chickens concurrent with a toe deformity ${ }^{13}$ and in otherwise unremarkable pet canaries. ${ }^{14}$ The rge (retinopathy globe enlarged) chicken develops posterior polar sub capsular cataracts. ${ }^{15}$ Toxic cataract genesis has been recorded in birds after nitrophenol administration ${ }^{16}$ and birds raised in continuous light suffer from lens opacification in addition to retinal degeneration and globe enlargement. ${ }^{17}$ Severe blinding cataracts occurred in ducks suffering from salt toxicosis ${ }^{18}$ and cataracts have also been recorded in birds infected with avian encephalomyelitis virus ${ }^{19}$ although there was also a genetic component to susceptibility to this agent. ${ }^{20}$ In addition cataract has been seen in owl eyes where other chronic lesions are a result of acute trauma and so may be postulated to be caused by high velocity blunt impact. ${ }^{21}$

Given this confusing panoply of aetiopathologies of avian cataract, investigation of the possible causes of cataracts in these corvids centred around the 5 Ds enumerated by Robman and Taylor in 2002: daylight, diet, diabetes, drugs and don't know! ${ }^{22}$ Daylight, and particularly ultraviolet light, is important because photo-oxidation of thiol groups to disulphide bridges in lens proteins can lead to their aggregation and subsequent cataract. Diet is important because ingested antioxidants can reduce this oxidative deterioration in the lens. To these five we should add DNA, since genes certainly impact on the likelihood for age-related as well as congenital cataract in $\operatorname{man}^{23}$ and the $\operatorname{dog}^{24}$ to name but two species.

\section{Materials and methods}

\section{Birds}

The birds involved in these studies were Western scrub jays, Aphelocoma californica) taken as fledglings from nests in Northern California and transported by air to the UK individually. Birds were housed in pairs cages measuring $2 \mathrm{~m}$ wide by $1 \mathrm{~m}$ high by $1 \mathrm{~m}$ deep, apart from days when they were being assessed when they were housed singly in $1 \mathrm{~m}$ by $1 \mathrm{~m}$ by $1 \mathrm{~m}$ cages and were maintained inside on a 12 hour light: 12 hour dark cycle with overhead $40 \mathrm{~W}$ incandescent strip lighting. They were fed on a diet of mixed nuts, dog biscuits, various fruits, wax worms and mealworms. During each behaviour test birds are housed singly in the experimental housing for a maximum of six hours during the day. In most cases the behaviour study is no more than one hour of caching behaviour. To facilitate food caching food restriction may be used for periods of no more than 4 daylight hours in one 24 hour period. The birds were weighed monthly, this initially requiring capture with nets, a technique which necessarily involved occasional trauma to the birds as they impacted on the wire netting sides of the aviaries during capture. Later in the study the frequency of catching to weigh the birds was reduced, as detailed further below.

\section{Ophthalmological examination techniques}

All birds were examined with direct and indirect ophthalmoscopy using Welch Alynn direct ophthalmoscope (Welch Allyn (UK) Ltd Aston Abbotts, UK) and Keeler Vantage indirect ophthalmoscope (Keeler, Windsor UK). Ocular lesions were documented photographically using a Coolpix 4500 camera (Nikon, Tokyo, Japan) for adnexal and ocular surface lesions ((Figures 2 and 4 show examples) and a Genesis D fundus camera (Kowa, Nagoya, Aichi, Japan) for more specific close-up documentation of lens lesions (as shown in Figure 3). Slit lamp biomicroscopy to document lens changes $^{25}$ was achieved using a Kowa SL14 handheld slit lamp
((Kowa, Nagoya, Aichi, Japan)). Cataracts were scored from absent (0) through individual vacuoles and small areas of lens opacity (1), intermediate areas covering less than half of the lens (2), more severe but no total cataract (3) and fully mature cataract involving the whole lens (4). Both eyes were examined separately and scored independently. Intraocular pressure was measured in each bird with a Tonovet rebound tonometer (ICare, Vantaa, Finland) and birds in which a lower than normal intraocular pressure (below $15 \mathrm{mmHg}$ ) was noted, indicating intraocular inflammation through a prostaglandinmediated increase in unconventional aqueous drainage, were treated with topical predinolone acetate anti-inflammatory drops. Behaviors of the birds were determined by observation of the birds in their enclosed to determine if they were able to negotiate their environments and apprehend food items but such behaviors were not quantified in this study.

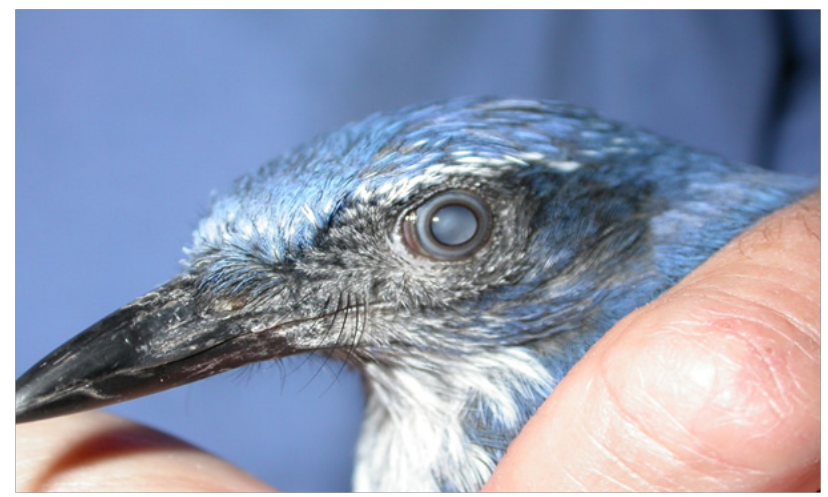

Figure $\mathbf{2}$ The index case - an ageing scrub jay with developing cataract (score 3).

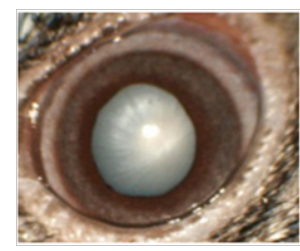

$3 \mathrm{a}$

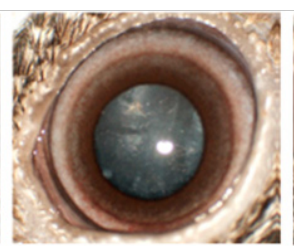

$3 b$

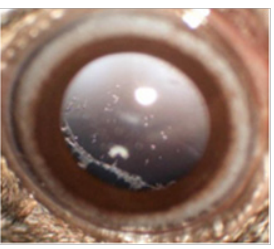

$3 c$
Figure 3 a. Cataracts in scrub jays a mature blinding opacity (score 4) b. developing cataract (score 2) c. incipient cataract vacuoles (score I).

\section{Results}

In 2004 the senior author was asked to examine a scrub jay from the colony with developing cataract. The bird had a substantial but not yet total opacity in both eyes (Figure 2) and, as it was one of the older birds in the colony (10-12 years of age), it was assumed that this was an age-related cataract. One year later an inspection showed a number of the birds to have some degree of lens opacity when examined with slit lamp biomicroscopy. A full examination of all birds was arranged and of the twenty two birds in the colony sixteen were diagnosed with some degree of cataract bilaterally, two had a unilateral cataract and only four had completely clear lenses. While these birds were among the older members of the colony, (between 6 and 12 years of age) they were not nearing the maximum projected longevity of this species in the wild at 15-20 years ${ }^{26}$ Two of the birds had mature cataract severe enough to render them blind and these birds, showing behavioral signs consistent with blindness (reduced ease of food prehension, loss of caching ability) were euthanased. Most birds had immature cataract, i.e. opacity which did not involve the entire lens with vision retained and no behavioral changes noted. 
Over two and a half years the birds were monitored and eleven birds showed increase in lens opacity and two with clear lenses developed cataracts. Two birds were euthanased because of blindness. A complicating issue was that during this period eight birds died of an unrelated haemorrhagic gastroenteritis, probably associated with reovirus infection and thus progression of their ocular signs could not be followed. New birds entering the colony were also examined during their period in quarantine. These individuals were thirty two wild-born young birds examined immediately after entry to the UK by air from California, during which flights they were transported in small $50 \mathrm{~cm} \times 50 \mathrm{~cm} \times 50 \mathrm{~cm}$ cages. Twelve birds had minute incipient or developing lens opacities at this early time point most of them being vacuolar changes often in linear arrangements (Figure 3).

In three birds cataract development appeared to be associated with a substantial intraocular inflammation with signs of blepharospasm, conjunctival hyperaemia and iridal swelling and darkening (Figure 4) together with reduced intraocular pressure as measured with a rebound tonometer, these signs often manifest after trauma associated with catching up of the bird. Correlation of these signs of inflammation with extent of cataract was not complete. Birds such as the one shown in figure $4 \mathrm{a}$, for instance, showed substantial intraocular inflammation but with only relatively early cataract, here as a linear opacity across the lens but in several birds these inflammatory signs after capture associated trauma allowed an association between cataract and trauma to be postulated. Catching the birds was reduced to once every three months after this point and the birds were weighed using a perch attached to a set of scales, thus reducing the possibility for catch-up trauma.

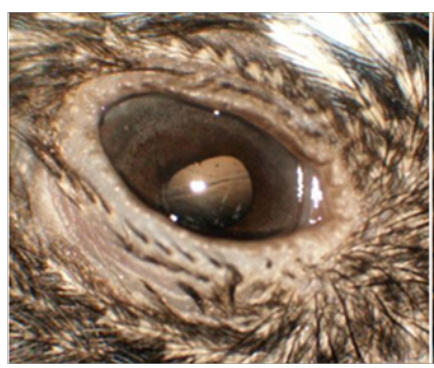

$4 \mathrm{a}$

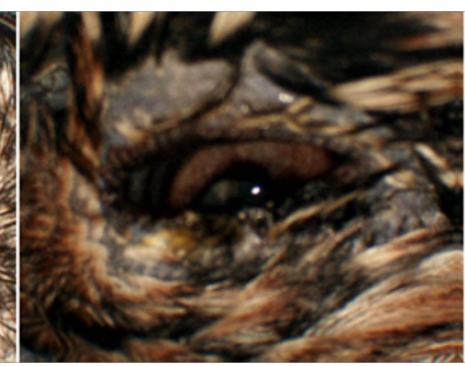

$4 b$
Figure 4 a. Uveitis in a scrub jay with cataract, b. Blepharospasm in a Eurassian jay.

The number of new cataracts, progressing cataracts and unchanged lenses, graded from zero (clear lens) to 4 (total mature cataract). In the 4 years covered by these examinations, during the period during which capture was reduced, the incidence of cataracts and number of cataractous lenses with increasing opacities reduced significantly, as shown by the change in gradient of the line in figure 5, documenting the increase in mean cataract score over time during monthly catch-up and three monthly catch-up (Figure 5 white box). The gradient of the trendline associated with cataract score is substantial in the first period of the study while the birds were being regularly caught, and virtually flat in the period after catch-up frequency was reduced, suggesting that ocular damage during capture was an important causative factor in cataract formation and progression.

Intraocular pressures in birds with clear lenses were $10.0 \pm 1.6 \mathrm{mmHg}$ (mean \pm standard deviation) while those with cataracts had a lower pressure of $8.1 \pm 1.1 \mathrm{mmHg}$, these significantly different at $\mathrm{p}<0.01 \mathrm{using}$ a Student's T test, while those with overt uveitis has an intraocular pressure of $7.0 \mathrm{mmHg}$, again significantly lower than normal eyes at $\mathrm{p}<0.01$ but not significantly different from those with cataracts $(\mathrm{p}=0.12)$. Other corvid species in Professor Clayton's research colony such as the jackdaw (Corvus monedula) and Eurassian jay (Garrulus glanderous) also appeared to be affected on occasion by these acute inflammatory changes but to a lesser degree by the ongoing lens changes seen in the scrub jays.

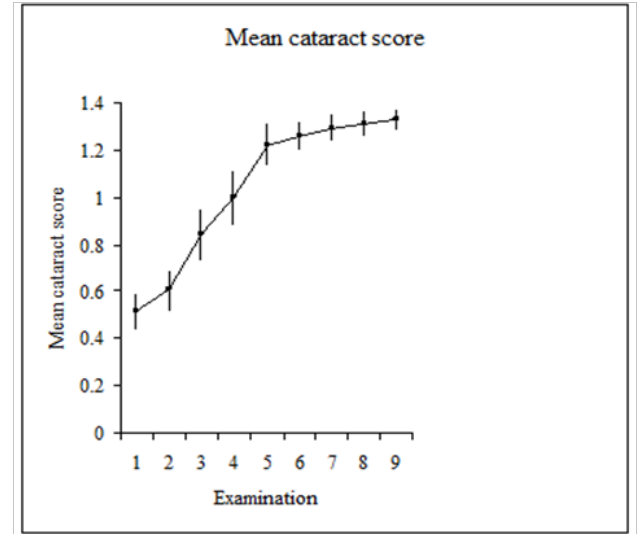

Figure 5 Mean cataract score of all scrub-jays during period of frequent capture (black box) and reduced capture frequency (white box), examinations of all birds being undertaken every six months.

\section{Discussion}

As noted in the introduction, a number of factors can be important in cataract formation. Of the 5 Ds of daylight, diet, diabetes, drugs and don't know as inumbrated by Taylor, drugs and diabetes can be discounted in these birds, but light irradiance and diet may be significant. The birds were kept inside so daylight, and in particular UV light as a cataractogenic factor can be excluded. The diet of wild scrub jays is wide from insects to fruits. ${ }^{26}$ An initial diet of Harrison's bird mix given in the early months of the development of this colony may have been insufficient in anti-oxidants although this is unlikely given the supplementation the diet provides. The diet given to the birds contained corn, hull-less barley, hulled gray millet, toasted soybeans, peas, lentils, peanut kernels, sunflower kernels, toasted oat groats, alfalfa, brown rice, chia, calcium carbonate, bentonite, mixed tocopherols (as a source of vitamin E), sea kelp, salt algae meal, vitamin A palmitate, vitamin D3 supplement, dl-alpha tocopheryl acetate, vitamin B12, riboflavin, calcium pantothenate, niacin, pyridoxine hydrochloride, biotin, thiamine mononitrate, folic acid, zinc sulfate, manganese sulfate, copper sulfate, sodium selenite, calcium carbonate and sunflower oil. ${ }^{27}$ This diet is specifically manufactured to avoid the deficiencies caused by feeding a solely seed-based diet. Nevertheless the diet of these captive corvids was widened to include fruits, vegetables and mealworms, but this wide dietary supplementation did not prevent cataract formation or progression. It could be said that a specific dietary factor might have been missed, but given that many thousands of birds internationally are fed on this mix without cataract formation it is unlikely that a dietary actor played a part in this ocular condition in these bids, The question of whether these birds were genetically susceptible to cataract was impossible to answer definitively. The fact that these were wild caught animals and that cataract has not been detected in wild individuals previously tends to negate an inherited cause. The fact that even young birds (less than 4 months) examined within days after capture and transport had the earliest of lens opacities and that the vacuoles were irregular cortical 
changes suggested that a genetic cause was unlikely. The lack of observed cataract in the wild population of these birds again argues against an inherited cause, although it may be that affected birds would not survive in the wild and thus would not be observed in the living population.

The association of some cataracts with other signs of ocular trauma, together with the likelihood of injury during frequent capture suggested a further D - that of damage. Observation of birds during capture with nets showed that many fly into the wire netting which constitutes the aviary sides giving a significant possibility of eye damage during capture. To these authors minds the change in progression of lens opacities only at the point when capture frequency was reduced does suggest an association between capture and cataract is possible, although we understand that other hypotheses regarding the causes of lens opacification might be possible to surmise.

Loss of vision would be expected to be detrimental to these birds; as Emery ${ }^{3}$ notes 'Like primates, and in common with most other groups of birds, the corvids are also highly visual. In primates, the evolutionary switch to a diurnal lifestyle was probably accompanied by two developments: (i) a greater reliance on visual social communication and (ii) more complex social structures. These principles also apply to corvids, given that they are highly visual and diurnal, as well as having complex social lives.' We might then expect that blindness would impede the birds' ability to cope with their environment, physical and social and reduce their welfare substantially. There is however anecdotal evidence from the birds in this colony with near total cataract that suggests that these individuals can actually cope well in confinement on their own although they may be bullied when in a group of birds (Clayton peers comm. 2016) The five freedoms put forward by the Farm Animal Welfare Council in 1977 include the freedom from pain, injury or disease, and the freedom to perform natural behaviors. The birds were clearly not free from disease in this case but they did not appear from observations of their behaviour to be adversely affected by visual compromise unless it was complete. Given these observations no action was taken with regard to birds which were still visual. Nevertheless freedom from fear anxiety cannot be guaranteed in a bird with substantially affected visual function and so the decision was made to euthanase any bird that was completely blind or where behavioral observations suggested it was not coping with its visual disturbance, even when not total.

It might be argued that keeping such birds originally caught in the wild in an aviary is unethical but their dietary and health needs are, it could be said, better catered for than as a bird in the wild continually needing to forage for food, potentially being at risk from pathogens such as West Nile Virus and living a substantially shorter lifespan than those in captivity where longevities of up to 20 years are not unheard of.

This, of course, brings us to an important factor, so far not discussed. Perhaps scrub jays in the wild are affected by these ocular abnormalities quite as much as these birds in captivity. We do not have evidence for prevalence of cataracts in wild scrub jays; it might be assumed that a genetic lesion that produced visual compromise would not survive in a wild population, but if these birds were affected after their average breeding age, such a condition would not adversely affect their reproductive success. It has recently been reported that scrub jays may be under oxidative stress as a reproductive $\operatorname{cost}^{27}$ and it is conceivable that this could have an impact on lens opacification, although the apparent correlation between lessening the handling of the birds and a reduction in cataract progression would seem to argue against this as an aetiopathogenic factor.

In conclusion, while it is impossible to be certain that trauma is the key factor in the development of these opacities, the clinical features of the cataracts, the frequency of traumatic episodes and the reduction in progression of cataracts when capture was undertaken less frequently, suggest that this is indeed the key etiological agent in cataract development, one which has not heretofore been widely recognized in the field of cataract biology. The fact that the behaviour of these birds is exceptionally well recorded has allowed the researchers and the veterinary ophthalmologists involved in their care to determine that until totally blind, which occurs in only a very small number of birds, these lens changes appear to have little effect on the birds' welfare, kept as they are in aviaries with a plentiful food source and ample environmental enrichment through the studies in which they are involved. As a final more personal comment, the present authors have to say that Clayton and her co-workers treat the birds in a very caring manner, as if they were their own children, an interaction which can only benefit the birds themselves.

\section{Acknowledgements}

None.

\section{Conflict of interest}

The author declares no conflict of interest.

\section{References}

1. Pepperberg IM. In search of King Solomon's ring: cognitive and communicative studies of Grey parrot (Psittacus erithacus). Brain Behav Evol. 2002;59(1-2):54-67.

2. Clayton N, Emery N. Corvid cognition. Current Biology. 2005;15:R80R81.

3. Emery, NJ. Are corvids 'feathered apes'? Cognitive evolution in crows, jays, rooks and jackdaws. In: Watanabe S, editor. Comparative Analysis of Minds. Tokyo: Keio University Press; 2004. p. 181-213.

4. Salwiczek LH, Emery NJ, Schlinger B, et al. The development of caching and object permanence in Western scrub-jays (Aphelocoma californica) which emerges first? J Comp Psychol. 2009;123(3):295-303.

5. de Kort SR, Correia SP, Alexis DM, et al. The control of food-caching behavior by Western scrub-jays (Aphelocoma californica). J Exp Psychol Anim Behav Process. 2007;33(4):361-370.

6. Correia SP, Dickinson A, Clayton NS. Western scrub-jays anticipate future needs independently of their current motivational state. Curr Biol. 2007;17(10):856-861.

7. Raby CR, Alexis DM, Dickinson A, et al. Planning for the future by western scrub-jays. Nature. 2007;445(7130):919-992.

8. Lo WK, Kuck JF, Shaw AP, et al. The altricial pigeon is born blind with a transient glycogen cataract. Exp Eye Res. 1993;56(1):121-126.

9. Braga IS 3rd1, Oda K, Kikuchi T, et al. Congenital cataract in 1-day-old French Mallard ducklings. Avian Pathology. 2009;38:9-11.

10. Landman WJ, Dwars RM. A cataract outbreak in broiler breeders. Avian Pathology. 1998;27(2):209-212.

11. Shibuya K, Nunoya T, Tajima M, et al. Scleral ectasia associated with hereditary retinal dysplasia in a mutant strain of Japanese quail (Coturnix coturnix japonica). Avian Patho. 1997;26(1):53-61. 
12. Braga IS 3rd, Oda K, Kikuchi T, et al. A new inherited muscular disorder in Japanese quails (Coturnix coturnix japonica). Vet Pathol. 1995;32(4):351-360

13. Chmielewski NT, Render JA, Schwartz LD, et al. Cataracts and crooked toes in Brahma chickens. Avian Dis. 1993;37(4):1151-1157.

14. Slatter DH, Bradley JS, Vale B, et al. Hereditary cataracts in canaries. $J$ Am Vet Med Assoc. 1983;183(8):872-874.

15. Inglehearn CF, Morrice DR, Lester DH, et al. Genetic, ophthalmic, morphometric and histopathological analysis of the Retinopathy Globe Enlarged (rge) chicken. Mol Vis. 2003;9:295-300.

16. Gehring PJ, Buerge JF. The cataractogenic activity of 2,4-dinitrophenol in ducks and rabbits. Toxicol Appl Pharmacol. 1969;14(3):475-486.

17. Lauber JK, McGinnis J. Eye lesions in domestic fowl reared under continuous light. Vision Res. 1966;6(12):619-626.

18. Gordus AG, Shivaprasad HL, Swift PK. Salt toxicosis in ruddy ducks that winter on an agricultural evaporation basin in California. $J$ Wildl Dis. 2002;38(1):124-131.

19. Halpin FB. Opacity of the lens in fowl associated with exposure to the virus of infectious avian encephalomyelitis-a case report. Avian Dis. 1967;11:146-148.

20. Barber CW, Blow WL. A genetic influence on cataract formation among White Leghorn incrosses following an outbreak of avian encephalomyelitis. Avian Dis. 1963;7(4):495-500.
21. Williams DL, Gonzalez Villavincencio CM, Wilson S. Chronic ocular lesions in tawny owls (Strix aluco) injured by road traffic. Veterinary Record. 2006;159(50):148-153.

22. Robman L, Taylor H. External factors in the development of cataract. Eye (Lond). 2005;19(10):1074-1082.

23. Graw J. Congenital hereditary cataracts. International Journal of Developmental Biology. 2004;48:1031-1044.

24. Mellersh CS, Pettitt L, Forman OP, et al. Identification of mutations in HSF4 in dogs of three different breeds with hereditary cataracts. Vet Ophthalmol. 2006;9(5):369-378.

25. Kercheval DB, Terry JE. Essentials of slit lamp biomicroscopy. $J$ Am Optom Assoc. 1977;48(11):1383-1389.

26. Woolfenden GE, Fitzpatrick JW. The Florida Scrub Jay: Demography of a Cooperative Breeding Bird. New Jersey: Princeton University Press; 1984.

27. Heiss RS, Schoerch SJ. Oxidative cost of reproduction is sex specific and correlated with reproductive effort in a cooperatively breeding bird, the Florida Scrub Jay. Physiological and Biochemical Zoology: Ecological and Evolutionary Approaches. 2012;85(5):499-503. 